\title{
Advanced Multicompartment Diffusion MRI Models and Their Application in Multiple Sclerosis
}

\author{
(DD.A. Lakhani, (D) K.G. Schilling, (D). Xu, and (D)F. Bagnato
}

\begin{abstract}
SUMMARY: Conventional MR imaging techniques are sensitive to pathologic changes of the brain and spinal cord seen in MS, but they lack specificity for underlying axonal and myelin integrity. By isolating the signal contribution from different tissue compartments, newly developed advanced multicompartment diffusion MR imaging models have the potential to detect specific tissue subtypes and associated injuries with increased pathologic specificity. These models include neurite orientation dispersion and density imaging, diffusion basis spectrum imaging, multicompartment microscopic diffusion MR imaging with the spherical mean technique, and models enabled through high-gradient diffusion MR imaging. In this review, we provide an appraisal of the current literature on the physics principles, histopathologic validation, and clinical applications of each of these techniques in both brains and spinal cords of patients with MS. We discuss limitations of each of the methods and directions that future research could take to provide additional validation of their roles as biomarkers of axonal and myelin injury in MS.
\end{abstract}

ABBREVIATIONS: $A D=$ axial diffusivity; $D_{a x}=$ intra-axonal diffusivity; $D B S I=$ diffusion basis spectrum imaging; $F F=$ fiber fraction; IVF = isotropic volume fraction; $\mathrm{NDI}=$ neurite density index (also $\mathrm{V}_{\mathrm{ic}}, \mathrm{V}_{\mathrm{in}}$, or $\mathrm{f}_{\mathrm{icvf}}$ ); NODDI = neurite orientation dispersion and density imaging; ODI = orientation dispersion index; $\mathrm{RD}=$ radial diffusivity; $\mathrm{SMT}=$ spherical mean technique; $\mathrm{V}_{\mathrm{ax}}=$ intra-axonal volume fraction

M $\mathrm{S}$ is an inflammatory-degenerative disease of the CNS affecting approximately 2.3 million people, nearly 900,000 of whom are in the United States. ${ }^{1}$

Transient and focal inflammation with variable degrees of demyelination and axonal injury is the pathologic hallmark of MS lesions. The acute inflammatory phase resolves within a few weeks. ${ }^{2,3}$ Thereafter, some lesions undergo repair of injured myelin and axons, ${ }^{4}$ while others may fail to repair and evolve into chronic plaques featured by variable degrees of chronic demyelination and axonal injury. ${ }^{4}$ Axons do not survive chronic

Received November 16, 2019; accepted after revision February 3, 2020.

From the Neuroimaging Unit (D.A.L., F.B.), Neuroimmunology Division, Department of Neurology; Division of Internal Medicine (D.A.L.); and Department of Radiology and Radiological Sciences (K.G.S., J.X.), Vanderbilt University Institute of Imaging Sciences, Vanderbilt University Medical Center, Nashville, Tennessee; Department of Radiology (D.A.L.), West Virginia University, Morgantown, West Virginia; and Department of Neurology (F.B.), VA Tennessee Valley Healthcare System, Nashville, Tennessee.

This work was supported by the National Institutes of Health grants R01EB017230 and T32EB001628 (K.G.S.), the National Institutes of Health grant K25 CA168936 (J.X.), and the National MS Society grant PP-1801-29686 (F.B.).

Please address correspondence to Francesca Bagnato, MD, PhD, Neuroimaging Unit, Neuroimmunology Division, Department of Neurology, Vanderbilt University Medical Center, 2201 Children's Way, Suite 1222, Nashville, TN 37212; e-mail: francesca.r.bagnato@vanderbilt.edu

Indicates article with supplemental on-line tables.

Indicates article with supplemental on-line photo.

http://dx.doi.org/10.3174/ajnr.A6484 demyelination, leading to axonal atrophy, transection, and subsequent neuronal death. These neurodegenerative processes may also extend outside focal plaques to areas of normalappearing WM and normal-appearing $\mathrm{GM}^{5}$

Conventional MR imaging (T1WI and T2WI) is highly sensitive to focal tissue injury in MS but lacks specificity to axonal content. Furthermore, T1WI and T2WI do not capture disease processes outside focal lesions; hence, there remains a pressing need to identify an MR imaging biometric that is both sensitive and specific to lesional and nonlesional axonal injury and loss. ${ }^{6}$

Toward this effort, several advanced multicompartment diffusion-based models have been recently developed and applied in MS (On-line Figure). The advantage of these methods over the more commonly used DTI (On-line Figure $A$ ) is the ability to isolate the signal contribution from different tissue compartments, thereby increasing the specificity for tissue subtypes and associated injuries. These models include neurite orientation dispersion and density imaging (NODDI), diffusion basis spectrum imaging (DBSI), multicompartment microscopic diffusion MR imaging with the spherical mean technique (SMT), and several models enabled with high-gradient diffusion MR imaging.

While some of these methods are very close to clinical translation and even available on clinical scanners, others lag behind. The need for high-gradient diffusion MR imaging and relatively 
long scanning times is still an important limiting factor to a widespread application of some of these models.

In this review, we appraise the current literature on the physics principles, histopathologic validation, and clinical applications of each of these techniques in both brain and spinal cord imaging of patients with MS. We also discuss limitations of each of the methods and future directions for translational research to provide additional validation for their role as a proxy for axonal injury in MS.

\section{Neurite Orientation Dispersion and Density Imaging}

The NODDI model was developed to provide a clinically feasible technique for in vivo mapping of neurite orientation, dispersion, and density. ${ }^{7}$ The advantage of NODDI is the requirement of a moderate number of diffusion-weighted images acquired with only $2 \mathrm{~b}$-shells (b-values), in contrast to other multicompartment models that need large gradient strengths or extensive scan times. ${ }^{7}$

Principles of Physics. NODDI is a multicompartmental model that distinguishes 3 microstructural environments: intracellular (or intra-axonal), extracellular (or extra-axonal), and CSF compartments (On-line Figure $B$ ). By affecting diffusion in a unique way, each compartment results in a separate MR imaging signal.

The intracellular component is represented by the intradendrites and intra-axonal spaces. Due to the highly restricted nature of diffusion in this space, the intracellular component is modeled as a set of geometric "sticks," or infinitely thin cylinders, with diffusion completely restricted perpendicular to these sticks but unhindered along them. The diffusivity along a stick is assumed to be fixed at $1.7 \times 10^{-3} \mathrm{~mm}^{2} / \mathrm{s}$. This set of sticks can be arranged in many distributions of orientations, eg, all coherently aligned in a single direction or more dispersed and spread over a range of orientations. The distribution of neurites (ie, sticks) is described by an average direction and a concentration parameter, which describes the orientation dispersion around the main direction.

The extracellular component represents the space around the neurites or axons, which is occupied by glial cells and neuronal cell bodies. In contrast to the restricted intracellular space, the diffusion in this space is "hindered" by neurites and is modeled as a simple anisotropic Gaussian distribution (as in DTI). The parallel and perpendicular diffusivities of the tensor in extracellular space are physically determined by the neurite morphology itself (ie, the intrinsic diffusivity and the neurite dispersion).

The CSF compartment is modeled as isotropic Gaussian diffusion with a fixed isotropic diffusivity $\left(3.0 \times 10^{-3} \mathrm{~mm}^{2} / \mathrm{s}\right)$.

Thus, the NODDI model results in metrics describing orientation, shape, diffusivities, and fractions of the different compartments. As reported in On-line Table 1, NODDI produces a number of clinically relevant indices: 1) the intracellular volume fraction, represented by the neurite density index (referred to in the literature as NDI, $\mathrm{V}_{\mathrm{ic}}, \mathrm{V}_{\mathrm{in}}$, or $\mathrm{f}_{\mathrm{icvf}}$ ), that ranges from 0 to 1 , denoting complete loss or full preservation of axons, respectively; 2) the neurite orientation dispersion index (ODI), which ranges from 0 , representing perfectly coherently oriented WM structures, to 1 , representing isotropically dispersed neurites; and 3) the isotropic volume fraction (often abbreviated as IVF, $\mathrm{V}_{\text {iso }}$, or $\mathrm{f}_{\mathrm{iso}}$ ), which can be interpreted as the voxel volume fraction of free water (ie, CSF).

Fixing the intrinsic and isotropic diffusivities and deriving the extracellular diffusivities from the dispersion index reduces the number of free parameters and simplifies the fitting of the model. ${ }^{8-10}$

Histopathologic Validation. Grussu et $\mathrm{al}^{11}$ used the thoracic spine tissue of a patient with primary-progressive MS and a lumbar spine specimen of a patient with secondary-progressive MS, as well as of 2 healthy controls. The authors used circular variance as a histologic marker of neurite orientation variability, and the myelin staining fraction, astrocyte staining fraction, microglia staining fraction, and neurofilament staining fraction as markers of myelin, astrocytes, microglia, and axon contents, respectively. Correlations were seen between ODI and circular variance in the normal-appearing WM of both healthy controls $(r=0.84, P<.001)$ and patients with MS $(r=0.60, P=.001)$, as well as between ODI and the myelin staining fraction in MS samples $(r=0.40, P<.05)$. Negative correlations were seen between NDI and circular variance $(r=-0.78, P<.001)$ in healthy controls and between NDI and the myelin staining fraction $(r=0.74, P<.001)$, neurofilament staining fraction $(r=$ $0.56, P<.01)$, and the microglia staining fraction $(r=0.49$, $P<.01)$ in patients with MS. No significant correlations were observed between IVF and histology markers. The authors found similar but less specific results with DTI-derived indices, in that several DTI-derived metrics correlated with histopathologic counterparts of myelin injury (like NODDI) as well as astrocyte/microglia infiltrates. In addition, none of the DTI indices showed a correlation with circular variance. These key findings highlight the ability of NODDI to show a different layer of pathology not captured by conventional DTI, eg, changes in the complexity of neurite arborizations.

Clinical Applications. Several cross-sectional studies have been performed to test the clinical feasibility and the ability of NODDI to differentiate tissue injury in MS and its associations with patients' disabilities. NODDI acquisition has been reported as $\sim 22$ minutes for whole-brain imaging $\left(0.4 \times 0.4 \times 2 \mathrm{~mm}^{3}\right.$ resolution, 2 b-shells, and 90 directions) $)^{12}$ and $\sim 18$ minutes for a single section of the cervical spinal cord $(1.25 \times 1.25 \mathrm{~mm} \times 10$ $\mathrm{mm}^{3}, 2$ b-shells, and 96 directions). ${ }^{13}$

We detail significant clinical findings reported in all published clinical studies in On-line Table 2. In summary, it was noted that when reaching a statistically significant difference, $\mathrm{NDI} / \mathrm{V}_{\text {ic }}$ values were always lower in lesions compared with normal-appearing $\mathrm{WM},{ }^{12,14-16}$ in normal-appearing WM compared with the normal WM of healthy controls (both in brains $^{14,16,17}$ and spinal cord ${ }^{13,17}$ ), and in the spinal cord normal-appearing GM compared with the GM of healthy controls. ${ }^{13}$ These results were consistent across patients with relapsing-remitting $^{12-17}$ or secondary-progressive MS. $^{12,16,17}$ However, thus far, only values of NDI measured in both the cortex ${ }^{18}$ and the spinal cord ${ }^{17}$ were found to be associated with clinical disability measured with the Expanded Disability Status 
Scale ${ }^{19}$ in patients with both relapsing-remitting ${ }^{17,18}$ and secondary-progressive ${ }^{17,18}$ MS.

On the contrary, ODI measurements yielded more contradictory results because ODI measurements were higher in lesions compared with normal-appearing WM in some studies focused on patients with relapsing-remitting $\mathrm{MS}^{13,14,18}$ and lower in others focused on either relapsing-remitting or mixed MS populations. ${ }^{12,15}$ Similarly, while Schneider et $\mathrm{al}^{15}$ found lower levels of ODI in the brain normal-appearing WM of patients with relapsing-remitting MS; By et $\mathrm{al}^{13}$ reported opposite trends in the spinal cord of a similar population of patients. Only cortical ODI measures were associated with clinical decline in patients with both relapsing-remitting ${ }^{14,18}$ and secondary-progressive ${ }^{18}$ MS.

Summary of Advantages and Limitations. NODDI appears to offer a clinically feasible technique that adds a layer of pathologic sensitivity and specificity to the detection of MS disease. A high interstudy variability is observed with ODI, thus making its interpretation complex. This finding is not surprising because by depicting the degree of tissue organization, ODI is a more granular, and therefore variable, measure of disease variability. It is proposed that ODI is more informative if used to assess longitudinal changes of individual patients rather than to assess comparisons between patients. On the contrary, although not always sensitive to disease, NDI/ $\mathrm{V}_{\mathrm{ic}}$ appears to capture some degree of change in axonal content, which is also measurable in perilesional tissue.$^{16}$

A few technical limitations need to be acknowledged regarding this model: 1) NODDI simplifications limit the model to simple fiber geometries, 2) the fixed diffusivities may not be true in altered physiology of the disease, and may bias measurements, and 3) debate remains on the relationship (and absolute values) of the intrinsic intra- and extracellular diffusivities. ${ }^{8-10}$

Thus, further work is needed to untangle these technical challenges, and to investigate the efficacy of NODDI-derived metrics as biomarkers of neurodegeneration, by assessing the sensitivity to tissue injury and clinical measures in larger cohorts of patients followed longitudinally over time.

\section{Multicompartment Microscopic Diffusion MR Imaging with the Spherical Mean Technique}

The SMT estimates microscopic features specific to the intra- and extraneurite compartments in the CNS. The use of the spherical mean technique is the most relevant advantage of this method, in that the SMT minimizes the confounding effects derived from axonal fiber crossings, curving, and orientation dispersion. SMT is suitable for clinical applications that require information on axonal volume fraction as well as axonal directions if one obtains spherical deconvolution.

Principles of Physics. SMT requires $\geq 2$ b-shells (each with multiple diffusion directions) and assumes diffusion-weighted signals arising from intra- and extra-axonal spaces (On-line Figure $C$ ), resulting in the apparent intra-axonal volume fraction $\left(\mathrm{V}_{\mathrm{ax}}\right)$ and the apparent extra-axonal volume fraction. Instead of approximating the whole intra- and extra-axonal spaces using single diffusion tensors, SMT assumes that signals arise from many "peraxon" diffusion tensors in arbitrary directions. ${ }^{20}$ By averaging the same b-value diffusion MR imaging signals over all directions, one can remove the orientation dependence of signals. Moreover, SMT assumes the following: 1) the intra-axonal radial diffusivity (RD) is zero due to the very small axon sizes in the CNS, and 2) the axial diffusivity (AD) is the same in both intra- and extra-axonal spaces due to the lack of restriction per axon. By such means, SMT can separate intra- and extra-axonal signals and 2 independent microstructural parameters, ie, the $\mathrm{V}_{\mathrm{ax}}$ and the apparent intra-axonal diffusivity $\left(D_{a x}\right)$ can be fit from the data. Other microstructure parameters, such as extra-axonal $\mathrm{RD}$, can be calculated from $\mathrm{V}_{\mathrm{ax}}$ and $\mathrm{D}_{\mathrm{ax}}$ (On-line Table 1).

Histopathologic Validation. There are no studies in either humans with MS or animal models of MS that validated SMTderived metrics against histopathologic counterparts; however, there is histopathologic validation of SMT in other models of diseases. Kaden et $\mathrm{al}^{21}$ studied the mouse model of the tuberous sclerosis complex and evaluated microscopic features specific to intra- and extraneurite compartments. The tuberous sclerosis animal model is more suitable for detecting CNS axonal injury than the MS model of experimental autoimmune encephalomyelitis because it is free from the inflammatory component that contaminates data from experimental autoimmune encephalomyelitis. $V_{a x}$ and $D_{a x}$ were measured in several $W M$ tracts of the brain, including the genu, midbody, splenium of the corpus callosum, and anterior commissure in both tuberous sclerosis and control mice. $\mathrm{V}_{\mathrm{ax}}$ values were lower $(P<.05)$ and $\mathrm{D}_{\mathrm{ax}}$ values were higher $(P<.05)$ in the WM of animals with tuberous sclerosis compared with controls. Furthermore, a decrease in the myelinated axon fraction (with the myelin fraction excluded, $P<.001$ ) was mirrored by a reduction of the MR imaging-based $\mathrm{V}_{\mathrm{ax}}$. Although obtained with a different disease model, the provided validation against axonal histology is fundamental. It is applicable to any condition affecting myelin and axonal integrity and supports the ability of SMT to quantify axonal content without artifactual effects from fiber-crossing and orientation dispersion.

Clinical Application. Only 2 studies investigating the feasibility and applicability of SMT in the brains ${ }^{22}$ and spinal cords ${ }^{23}$ of patients with MS have been performed thus far. The authors reported acquisition times between $\sim 18$ minutes $^{23}$ (single-section C-spine, $1.25 \times 1.25 \times 10 \mathrm{~mm}$ resolution, $2 \mathrm{~b}$-shells, with 32 and 64 directions) and $\sim 22$ minutes (full brain coverage, $0.4 \times 0.4 \times$ $2 \mathrm{~mm}$ resolution, 90 directions). ${ }^{22}$ The implementation of a recently developed multiband technique can remarkably reduce the scan time while keeping similar image quality. For example, a multiband factor of 3 can reduce the total scan time of SMT from $\sim 22$ minutes to $\sim 10$ minutes.

In brains ${ }^{22}$ and spinal cords ${ }^{23}$ of patients with $\mathrm{MS}, \mathrm{V}_{\mathrm{ax}}$ and $\mathrm{D}_{\mathrm{ax}}$ (brain only ${ }^{22}$ ) differed between lesions and normal-appearing WM. Decreased values of $V_{a x}$ were also observed in normalappearing WM compared with normal WM of healthy controls (spinal cord only ${ }^{23}$ ). Reduction in acquisition schemes by $50 \%$ did not affect the sensitivity of SMT in distinguishing all 3 tissue subtypes. $^{23}$

Grussu et $\mathrm{al}^{24}$ studied the diffusion time-dependence of IVF, ODI, $\mathrm{V}_{\mathrm{ax}}$, and $\mathrm{D}_{\mathrm{ax}}$ in 3 healthy controls and quoted percentage 
changes of these parameters in the diffusion time range of 29$76 \mathrm{~ms}$. Increases in IVF $(4.1 \%)$ and $\mathrm{V}_{\mathrm{ax}}(2 \%)$ and decreases in the ODI $(-16 \%)$ and $\mathrm{D}_{\mathrm{ax}}(-5.4 \%)$ as a function of the diffusion time were noted in the motor columns of the spinal cord. Furthermore, increases in the IVF $(3.7 \%)$ and decreases in the ODI $(-15 \%), \mathrm{V}_{\mathrm{ax}}(-5.1 \%)$, and $\mathrm{D}_{\mathrm{ax}}(-11.5 \%)$ were measured in the WM of the sensory columns of the spinal cord. The results suggest a diffusion time-dependence of NODDI- and SMT-derived metrics, which will provide new opportunities to optimize these methodologies for spinal cord imaging.

Summary of Advantages and Limitations. By taking the averages of diffusion signals over all directions and making no assumptions about the orientation of fibers, SMT removes the orientation dependence of diffusion MR imaging signals. These elements simplify data analysis and improve the SNR because they remarkably reduce the interscan variability. Histopathologic evidence supports the ability of SMT to isolate signal contributions from axons. In clinical studies, both $\mathrm{V}_{\mathrm{ax}}$ and $\mathrm{D}_{\mathrm{ax}}$ have shown the potential to differentiate tissue subtypes in the brains and spinal cords of patients with MS. Even by reducing the acquisition time by $50 \%$, the SMT metrics were found to be equally sensitive in differentiating these tissue subtypes. ${ }^{23}$ Both metrics, however, have failed to show correlation with the clinical measure of disease. This issue may be related simply to the relatively small sample size of cross-sectional studies performed thus far. Larger studies with longitudinal designs are warranted to elucidate the role of SMTderived metrics as biomarkers of neurodegeneration in MS.

\section{Diffusion Basis Spectrum Imaging}

DBSI was proposed to resolve multiple tensor-like populations of water that may arise not only in healthy tissue but also from axon injury, inflammation, and demyelination. ${ }^{25}$ Despite solid histopathologic validation studies, clinical applications of this technique are limited.

Principles of Physics. DBSI models diffusion as a combination of multiple discrete anisotropic tensors and a spectrum of isotropic diffusion tensors (On-line Figure D). The discrete anisotropic tensors are intended to represent myelinated and unmyelinated axons oriented in varying directions. The isotropic tensors, then, represent the integration of multiple pools of water, typically separated into the restricted spectrum from $0 \leq$ apparent diffusion coefficient $\leq$ $0.3 \times 10^{-3} \mathrm{~mm}^{2} / \mathrm{s}$, which may reflect cellularity. The nonrestricted isotropic diffusion spectrum from $0.3 \times 10^{-3} \mathrm{~mm}^{2} / \mathrm{s}<$ apparent diffusion coefficient $<3.0 \times 10^{-3} \mathrm{~mm}^{2} / \mathrm{s}$ reflects extracellular edema and CSF. Thus, in DBSI, the diffusion signal is modeled as a summation of many diffusion tensors and an integration of free water with varying diffusivities, involves fitting many free variables, and uses a complex signal-fitting approach.

DBSI results in estimates of several anisotropic diffusion components (representing intra-axonal water molecules), each weighted by signal intensity fractions (referred to as their fiber ratios), oriented in the direction of the fiber population, and associated with their own parallel and perpendicular diffusivity. ${ }^{25}$ Thus, just like DTI, DBSI results in metrics of RD, AD, fractional anisotropy, and the fraction of anisotropic components (called the fiber fraction [FF]) (On-line Table 1). In addition, the fraction of water molecules of highly restricted isotropic structures can be measured (referred to as the restricted isotropic diffusion fraction or as the cell ratio in the original implementation), as well as that of the less restricted isotropic water (referred to as the water ratio).

Histopathologic Validation. Shirani et $\mathrm{al}^{26}$ studied histopathologic correlates of DBSI in comparison with DTI in a single tumefactive lesion of a patient with newly diagnosed MS. DTI-derived $\mathrm{AD}$ and $\mathrm{RD}$ and DBSI-derived RD were increased, while DTIand DBSI-derived fractional anisotropy were decreased in the lesion compared with the normal WM. DBSI-derived FF was also minimally decreased in the lesional area. Histopathologic analyses showed features of demyelination with increased inflammation and cellularity, but little axonal damage. Overall, the authors concluded that DBSI more selectively depicted demyelination and cellular increase and was not affected by the presence of edema, which, instead, biased the DTI-derived AD values.

Wang et $\mathrm{al}^{27}$ studied postmortem examinations of the cervical spinal cords of 2 patients with relapsing-remitting MS and 1 with primary-progressive MS. The authors found correlations between FF and silver stain $(r=0.7-0.8, P<.001$, in $3 / 3$ samples); DBSderived RD and Luxol fast blue stain $(r=-0.4--0.84, P<.05$, in $3 / 3$ samples); and restricted fraction and hematoxylin stain, reflecting the number of nucleated cells $(r=0.3-0.8, P<.05$, in $2 / 3$ samples). No significant correlations were seen between DBSI-derived AD and silver stain.

To assess the performance of DBSI and DTI metrics in the presence of CSF contamination, Wang et $\mathrm{al}^{27}$ compared values derived from regions in the center of the corpus callosum (representing pure axonal fibers) and from areas of the WM bordering the lateral ventricles (representing CSF contamination) in 5 healthy controls. DBSI-derived $\mathrm{AD}$ and $\mathrm{RD}$ for each of these voxels were indistinguishable, while CSF contamination substantially altered DTI-derived AD and RD.

Furthermore, DBSI and DTI values were derived from regions at the crossing between the corona radiata and the corpus callosum; and within the corona radiata and the corpus callosum in 5 healthy controls, to assess the effect of fiber-crossing. DBSI AD and RD values were similar, while DTI AD and RD values differed between the corpus callosum and regions at the crossing between the corpus callosum and the corona radiata. DBSI AD values did not differ between the pure corona radiata and corona radiata fibers extracted from the region of crossing with the corpus callosum.

In conclusion, contrary to DTI, DBSI-derived $\mathrm{AD}$ and $\mathrm{RD}$ values were not influenced by CSF contamination and fiber-crossing.

Clinical Application. Shirani et $\mathrm{al}^{28}$ applied DBSI in patients with relapsing-remitting MS ( $n=22$, disease duration $=2.0-18.8$ years), secondary-progressive MS ( $n=16$, disease duration $=15.4-39.0$ years), and primary-progressive MS $(n=17$, disease duration $=6.5-$ 18.3 years). They used recursive partitioning, a nonparametric decision tree-based regression and classification, to assess the ability of DBSIderived metrics to classify patients with different disease subtypes. FF and restricted isotropic fraction classified $35 / 55$ patients (63\%) into the 
correct MS subtype. Similarly, FF, nonrestricted isotropic fraction and $\mathrm{RD}$ measured in the normal-appearing WM of the corpus callosum correctly classified $37 / 55$ patients (67\%). No differences were seen in the $\mathrm{FF}$, isotropic restricted fraction, isotropic nonrestricted fraction, $\mathrm{RD}$ and $\mathrm{AD}$ between WM lesions and normal-appearing WM among patients with relapsing-remitting, secondary-progressive, and primary-progressive MS

Summary of Advantages and Limitations. The performance of DBSI has been extensively validated against histopathology in humans, and DBSI was shown to perform better than conventional DTI in the presence of edema. Accordingly, DBSI has shown promising results for assessing axonal quantity (DBSI-AD and -FF) while correcting for signal impurity derived from fluid contamination and fiber-crossing. Clinical applications of this methodology are in their infancy. Initial studies support the notion that DBSI correctly classifies the phenotype of about twothirds of patients with MS. Additional studies are necessary to further elucidate its role in vivo. Similarly, sensitivity and specificity validations are lacking in simulated and physical phantom environments, and further investigation of precision and bias is necessary.

\section{High-Gradient Diffusion MR Imaging}

Principles of Physics. This method is similar to the previously developed AxCaliber method, ${ }^{29}$ which maps the mean axon diameter and water fractions of axons and CSF. ${ }^{30}$ It assumes diffusion signals arising from 3 nonexchanging compartments: restricted diffusion in the intra-axonal space, hindered diffusion in the extra-axonal space, and free diffusion in CSF (On-line Figure E). Unlike NODDI and SMT, this method assumes that axons have nonzero diameters and typically require diffusion data with multiple diffusion times and multiple b-values. By assuming that axons are cylinders, analytic models can be fit to diffusion data to extract quantitative microstructural information, such as mean axon diameter and apparent intra-axonal volume fraction (On-line Table 1). Note that the obtained mean axon diameter is volume-weighted rather than an arithmetical mean value, the latter predominantly used previously in the pathology literature. ${ }^{30}$ It is challenging to fit comprehensive analytic equations to diffusion-weighted signal data from a voxel consisting of axonal fibers in $>1$ direction (such as fiber-crossing, curving, and dispersion). Therefore, this method was initially used only in regions with 1 dominant axonal fiber direction, such as the corpus callosum or the spinal cord. ${ }^{31,32}$ A recent report, however, has extended the application of this method to different WM tracts in the brain. ${ }^{33}$ This extended method assumes that axonal fibers are oriented in a principal direction, which makes it possible to average DWI signals perpendicular to this principal fiber orientation. By such a means, a volume-weighted axon diameter index can be obtained in all white matter tracts in the brain, as shown in On-line Figure $E$.

There are existing debates on the ability of this method to fit the axon diameter. Early studies of AxCaliber in vivo were found to overestimate axon diameters in the corpus callosum. ${ }^{30}$ Gradient strengths can definitely alleviate this issue, yet it is recognized that the reported mean axon diameters in the corpus callosum in healthy subjects are still overestimated compared with the histologic findings. ${ }^{31,34,35}$ Moreover, a recent study showed that the extra-axonal diffusion dominates the overall diffusion signals in the typical diffusion time range; thus, it should be used with caution to interpret the fitted axon diameter in vivo. $^{36}$ The high-gradient diffusion method requires special gradient coils with gradient strengths up to $300 \mathrm{mT} / \mathrm{m}$, which is much stronger than the $30-80 \mathrm{mT} / \mathrm{m}$ gradient strength on typical commercial MR imaging systems. This requirement is an important obstacle to clinical translation.

Clinical Application. Huang et $\mathrm{al}^{31,37}$ studied the clinical feasibility and applicability of high-gradient diffusion MR imaging in MS. The authors reported a scanning time of $\sim 51$ minutes for a whole-brain acquisition at a 2-mm isotropic resolution. ${ }^{37}$ Six patients with relapsingremitting MS (disease duration, 1-11 years) and 6 healthy controls were initially imaged with the aim of characterizing axonal disease in MS lesions and normal-appearing WM. Increased axonal diameter, decreased restricted fraction, increased free water fraction, and reduced mean axon density were described in lesions compared with normalappearing $\mathrm{WM}$, but not between normal-appearing WM and normal WM. $^{31}$

Recently, the same group ${ }^{37}$ reported data from a larger patient population, illustrated in On-line Table 2. Similarly, Santis et $\mathrm{al}^{38}$ found increased axonal diameters and decreased restricted volume fraction/axonal density in lesions compared with the normal-appearing WM of the corpus callosum, in normal-appearing WM compared with normal WM, as well as in the normalappearing WM of patients with secondary-progressive MS compared with that of patients with relapsing-remitting MS.

Summary of Advantages and Limitations. Clinical studies show the potential for high-gradient diffusion MR imaging to differentiate tissues with different degrees of axonal pathology. The physics underlying the use of high-gradient diffusion MR imaging limit its ability to quantify axon diameter in the presence of fiber-crossing. Furthermore, no histopathologic validation has been performed, a factor that currently hampers extrapolating the full significance from the in vivo data. It is interesting to note that the axonal diameter measured using this technique were larger in patients as compared to that of healthy controls. The finding may reflect the well-known pathologic course of axon death. ${ }^{39}$ Axons undergo remodeling with initial swelling before atrophy and cell death. Thus, the time when patients are imaged may determine the direction of group differences one can find.

\section{CONCLUSIONS}

There is an urgent need to discover a biometric of neurodegeneration and repair, which can be used to untangle MS disease progression before it manifests as an irreversible disability. Advanced, multi-shellbased diffusion methods have the potential to add pathologic specificity to imaging findings, and hence serve as biomarker of neurodegeneration and neuroregeneration. Additional work is required, however, to translate these methods to clinical application.

Most of these techniques have 2 main technical advantages. First, they use conventional, off-the-shelf diffusion MR imaging pulse sequences, which are widely available on clinical MR imaging 
scanners. This use removes any obstacle toward their translation to routine clinical imaging. Second, most of these diffusion methods have their data analysis codes open to the public, making it easy to implement independent studies. However, technical improvements based on the implementation of multibands are warranted to enhance clinical reliability. ${ }^{40,41}$ These changes will aim for faster acquisitions with higher signal-to-noise ratio. Effort will also be devoted to the implementation of postprocessing techniques for the spinal cord and the development of atlases that can more accurately couple these advanced imaging metrics with anatomic landmarks.

From a histopathologic validation standpoint, the provided evidence is solid but remains limited because it is based on a small number of samples or animal studies using a different model of neurodegeneration. Ideally, postmortem studies on human brains are the closest ones for studying human disease. These studies, however, have the limitations of formalin fixation, which substantially alters diffusivity properties and shortens the $\mathrm{T} 1$ and $\mathrm{T} 2$ relaxation times of tissues compared with their in vivo state. ${ }^{42}$ Furthermore, there is a time-dependent effect of formalin fixation on diffusivity. Data show that scanning different brains at a consistent postfixation interval leads to different absolute measurements between samples, which could, in turn, lead to erroneous conclusions. ${ }^{43}$

Histopathologic validation of lesions requiring biopsy is also an efficient method but has the caveat that lesions undergoing biopsy are usually atypically aggressive. While not resembling, in full, MS pathology, these evaluations still offer a solid validation method for the imaging-pathologic correlates. Similarly, animal studies, while again offering a disease model that may not resemble, in full, that of MS, do offer opportunities to validate histologic counterparts of imaging data. It is unlikely, however, that individual centers will be able to offer a full spectrum of validation; and to this end, collaborative efforts are certainly crucial to delineate the solidity and reproducibility of individual findings.

From a clinical standpoint, it appears that metrics derived from each of the discussed techniques are sensitive to different degrees of pathology in MS for both the brain and spinal cord. A common criticism of those methods is that changes measured in different tissue types using metrics derived from advanced diffusion models may, at times, be like those measured using conventional DTI or other quantitative techniques. Even if the criticism is valid, given the physics principles underpinning each of those techniques, advanced multicompartment diffusion MR imaging models offer a novel and superior way to visualize and quantify pathology. Thus, even if the effect size is similar, all these models add a substantial layer of pathologic specificity to disease detection.

Whether this increased pathologic specificity corresponds to an improved ability to explain disability remains to be assessed. While data thus far do not seem to necessarily suggest so, none of the presented studies have been powered to address this question. It is also likely that across-time changes of these proposed measures, rather than singletime-point assessments, are reflective of across-time disease progression. Additional larger clinical studies to address this question are the next step. It is indeed likely, and most relevant clinically, that individual patient changes across time, rather than between-subject cross-sectional comparisons, are going to be both more informative and explicative of the pathologic and clinical evolution of MS. This consideration is particularly relevant in lieu of the fact that axonal size and volume change with time as a function of the pathologic processes. Swelling and enlargement often at times precede shrinkage and transection, ${ }^{5,39}$ and this feature must be taken into consideration when comparing data from different patient cohorts or even when comparing patients with heathy controls in cross-sectional evaluations.

Last, but no less important, intercenter reliability measures must be estimated. Lack of intercenter and interscanner consistency is an important drawback and will consequently hinder clinical translation of any biomarker.

Future research based on cooperative effort aiming to fill the gap in the histopathologic, clinical, and technical knowledge as detailed above is most warranted.

\section{ACKNOWLEDGMENTS}

The authors would like to thank Dr Seth Smith for the design and conceptualization of the On-line Figure and Dr Karina Ciccone for thoroughly reviewing the manuscript for grammar and appropriateness of the delivered message.

Disclosures: Francesca Bagnato_UNRELATED: Speaker honoraria from EMD-Serono and Novartis. She serves/ed as site PI of multicenter studies sponsored by EMD- Serono and Novartis. Paid to the individual.

\section{REFERENCES}

1. Wallin MT, Culpepper WJ, Campbell JD, et al; US Multiple Sclerosis Prevalence Workgroup. The prevalence of MS in the United States: a population-based estimate using health claims data. Neurology 2019;92:e1029-40 CrossRef Medline

2. Bagnato F, Jeffries N, Richert ND, et al. Evolution of T1 black holes in patients with multiple sclerosis imaged monthly for 4 years. Brain 2003;126:1782-89 CrossRef Medline

3. Cotton F, Weiner HL, Jolesz FA, et al. MRI contrast uptake in new lesions in relapsing-remitting MS followed at weekly intervals. Neurology 2003;60:640-46 CrossRef Medline

4. Patrikios P, Stadelmann C, Kutzelnigg A, et al. Remyelination is extensive in a subset of multiple sclerosis patients. Brain 2006;129:3165-72 CrossRef Medline

5. Frischer JM, Bramow S, Dal-Bianco A, et al. The relation between inflammation and neurodegeneration in multiple sclerosis brains. Brain 2009;132:1175-89 CrossRef Medline

6. Fox RJ, Thompson A, Baker D, et al. Setting a research agenda for progressive multiple sclerosis: the International Collaborative on Progressive MS. Mult Scler 2012;18:1534-40 CrossRef Medline

7. Zhang H, Schneider T, Wheeler-Kingshott CA, et al. NODDI: practical in vivo neurite orientation dispersion and density imaging of the human brain. Neuroimage 2012;61:1000-16 CrossRef Medline

8. Hutchinson EB, Avram AV, Irfanoglu MO, et al. Analysis of the effects of noise, DWI sampling, and value of assumed parameters in diffusion MRI models. Magn Reson Med 2017;78:1767-80 CrossRef Medline

9. Kunz N, da Silva AR, Jelescu IO. Intra- and extra-axonal axial diffusivities in the white matter: which one is faster? Neuroimage 2018;181:314-22 CrossRef Medline

10. Lee HH, Fieremans E, Novikov DS. What dominates the time dependence of diffusion transverse to axons: intra- or extra-axonal water? Neuroimage 2018;182:500-10 CrossRef Medline

11. Grussu F, Schneider T, Tur C, et al. Neurite dispersion: a new marker of multiple sclerosis spinal cord pathology? Ann Clin Transl Neurol 2017;4:663-79 CrossRef Medline 
12. Chen A, Franco G, Smith S, et al. Measuring white matter axonal injury in patients with multiple sclerosis. In: Proceedings of the American Academy of Neurology Annual Meeting, Philadelphia, Pennsylvania. May 4-10, 2019

13. By $\mathrm{S}, \mathrm{Xu}$ J, Box BA, et al. Application and evaluation of NODDI in the cervical spinal cord of multiple sclerosis patients. Neuroimage Clin 2017;15:333-42 CrossRef Medline

14. Granberg T, Fan Q, Treaba CA, et al. In vivo characterization of cortical and white matter neuroaxonal pathology in early multiple sclerosis. Brain 2017;140:2912-26 CrossRef Medline

15. Schneider T, Brownlee W, Zhang H, et al. Sensitivity of multi-shell NODDI to multiple sclerosis white matter changes: a pilot study. Funct Neurol 2017;32:97-101 CrossRef Medline

16. Mustafi SM, Harezlak J, Kodiweera C, et al. Detecting white matter alterations in multiple sclerosis using advanced diffusion magnetic resonance imaging. Neural Regen Res 2019;14:114-23 CrossRef Medline

17. Collorone S, Cawley N, Grussu F, et al. Reduced neurite density in the brain and cervical spinal cord in relapsing-remitting multiple sclerosis: a NODDI study. Mult Scler 2019 Nov 4. [Epub ahead of print] CrossRef Medline

18. Spanò B, Giulietti G, Pisani V, et al. Disruption of neurite morphology parallels MS progression. Neurol Neuroimmunol Neuroinflamm 2018;5:e502 CrossRef Medline

19. Kurtzke JF. Rating neurologic impairment in multiple sclerosis: an Expanded Disability Status Scale (EDSS). Neurology 1983;33:144452 CrossRef Medline

20. Kaden E, Kruggel F, Alexander DC. Quantitative mapping of the per-axon diffusion coefficients in brain white matter. Magn Reson Med 2016;75:1752-53 CrossRef Medline

21. Kaden E, Kelm ND, Carson RP, et al. Multi-compartment microscopic diffusion imaging. Neuroimage 2016;139:346-59 CrossRef Medline

22. Bagnato F, Franco G, Li H, et al. Probing axons using multi-compartmental diffusion in multiple sclerosis. Ann Clin Transl Neurol 2019;6:1595-1605 CrossRef

23. By S, Xu J, Box BA, et al. Multi-compartmental diffusion characterization of the human cervical spinal cord in vivo using the spherical mean technique. NMR Biomed 2018;31:e3894 CrossRef Medline

24. Grussu F, Ianuş A, Tur C, et al. Relevance of time-dependence for clinically viable diffusion imaging of the spinal cord. Magn Reson Med 2019;81:1247-64 CrossRef Medline

25. Wang Y, Wang Q, Haldar JP, et al. Quantification of increased cellularity during inflammatory demyelination. Brain 2011;134:35903601 CrossRef Medline

26. Shirani A, Sun P, Schmidt RE, et al. Histopathological correlation of diffusion basis spectrum imaging metrics of a biopsy-proven inflammatory demyelinating brain lesion: a brief report. Mult Scler 2019;25:1937-41 CrossRef

27. Wang $Y$, Sun $P$, Wang $Q$, et al. Differentiation and quantification of inflammation, demyelination and axon injury or loss in multiple sclerosis. Brain 2015;138:1223-38 CrossRef Medline

28. Shirani A, Sun P, Trinkaus K, et al. Diffusion basis spectrum imaging for identifying pathologies in MS subtypes. Ann Clin Transl Neurol 2019;6:2323-27 CrossRef Medline
29. Assaf Y, Blumenfeld-Katzir T, Yovel Y, et al. AxCaliber: a method for measuring axon diameter distribution from diffusion MRI. Magn Reson Med 2008;59:1347-54 CrossRef

30. Alexander DC, Hubbard PL, Hall MG, et al. Orientationally invariant indices of axon diameter and density from diffusion MRI. Neuroimage 2010;52:1374-89 CrossRef Medline

31. Huang SY, Tobyne SM, Nummenmaa A, et al. Characterization of axonal disease in patients with multiple sclerosis using highgradient-diffusion MR imaging. Radiology 2016;280:244-51 CrossRef Medline

32. Duval T, McNab JA, Setsompop $\mathrm{K}$, et al. In vivo mapping of human spinal cord microstructure at 300mT/m. Neuroimage 2015;118:494-507 CrossRef Medline

33. Huang SY, Tian Q, Fan Q, et al. High-gradient diffusion MRI reveals distinct estimates of axon diameter index within different white matter tracts in the in vivo human brain. Brain Struct Funct 2019 Sep 28. [Epub ahead of print] CrossRef Medline

34. Huang SY, Nummenmaa A, Witzel T, et al. The impact of gradient strength on in vivo diffusion MRI estimates of axon diameter. Neuroimage 2015;106:464-72 CrossRef Medline

35. Aboitiz F, Scheibel AB, Fisher RS, et al. Fiber composition of the human corpus callosum. Brain Res 1992;598:143-53 CrossRef Medline

36. Burcaw LM, Fieremans E, Novikov DS. Mesoscopic structure of neuronal tracts from time-dependent diffusion. Neuroimage 2015;114:18-37 CrossRef Medline

37. Huang SY, Fan Q, Machado N, et al. Corpus callosum axon diameter relates to cognitive impairment in multiple sclerosis. Ann Clin Transl Neurol 2019;6:882-92 CrossRef Medline

38. Santis SD, Herranz E, Treaba CA, et al. Whole brain in vivo axonal diameter mapping in multiple sclerosis. In: Proceedings of the International Engineering in Medicine and Biology Conference, Berlin, Germany. July 23-27, 2019; 204-07

39. Kutzelnigg A, Lucchinetti CF, Stadelmann C, et al. Cortical demyelination and diffuse white matter injury in multiple sclerosis. Brain 2005;128:2705-12 CrossRef Medline

40. Feinberg DA, Setsompop K. Ultra-fast MRI of the human brain with simultaneous multi-slice imaging. J Magn Reson 2013;229:90100 CrossRef Medline

41. Veraart J, Novikov DS, Christiaens D, et al. Denoising of diffusion MRI using random matrix theory. Neuroimage 2016;142:394-406 CrossRef Medline

42. Shepherd TM, Thelwall PE, Stanisz GJ, et al. Aldehyde fixative solutions alter the water relaxation and diffusion properties of nervous tissue. Magn Reson Med 2009;62:26-34 CrossRef Medline

43. Shatil AS, Uddin MN, Matsuda KM, et al. Quantitative ex vivo MRI changes due to progressive formalin fixation in whole human brain specimens: longitudinal characterization of diffusion, relaxometry, and myelin water fraction measurements at 3T. Front Med (Lausanne) 2018;5:31 CrossRef Medline

44. Cutter GR, Baier ML, Rudick RA, et al. Development of a multiple sclerosis functional composite as a clinical trial outcome measure. Brain 1999;122:871- 82 CrossRef Medline 